\title{
Narrative review of frameworks for translating research evidence into policy and practice
}

\author{
Andrew J Milata,b,d and Ben Lic \\ a NSW Ministry of Health, Sydney, Australia \\ b Sydney Medical School, University of Sydney, NSW, Australia \\ c McMaster University, Ontario, Canada \\ ${ }^{d}$ Corresponding author: amila@doh.health.nsw.gov.au
}

\section{Article history}

Publication date: February 2017

Milat AJ, Li B. Narrative review of frameworks for translating research evidence into policy and practice. Public Health Res Pract. 2017;27(1):e2711704. doi: http://dx.doi. org/10.17061/phrp2711704

\section{Key points}

- Conceptual models provide a framework to describe and measure the research translation process

- Conceptual models for research translation are interpreted and applied by different health fields in different but related ways

- The most frequently applied knowledge translation framework in the literature was RE-AIM, followed by the knowledge translation continuum or ' $T$ ' models, and the Knowledge to Action framework

- A better understanding of how research translation frameworks are being used in practice will help identify the key barriers to effective research translation

\section{Abstract}

Background: A significant challenge in research translation is that interested parties interpret and apply the associated terms and conceptual frameworks in different ways. The purpose of this review was to: a) examine different research translation frameworks; b) examine the similarities and differences between the frameworks; and c) identify key strengths and weaknesses of the models when they are applied in practice.

Methods: The review involved a keyword search of PubMed. The search string was (translational research OR knowledge translation OR evidence to practice) AND (framework OR model OR theory) AND (public health OR health promotion OR medicine). Included studies were published in English between January 1990 and December 2014, and described frameworks, models or theories associated with research translation.

Results: The final review included 98 papers, and 41 different frameworks and models were identified. The most frequently applied knowledge translation framework in the literature was RE-AIM, followed by the knowledge translation continuum or ' $T$ ' models, the Knowledge to Action framework, the PARiHS framework, evidence based public health models, and the stages of research and evaluation model.

Conclusion: The models identified in this review stem from different fields, including implementation science, basic and medical sciences, health services research and public health, and propose different but related pathways to closing the research-practice gap.

\section{Introduction}

The generation and deployment of evidence in healthcare is an evolving field, and bridging the 'gap' between research evidence and the complexities of policy and practice is an ongoing challenge. ${ }^{1}$ In 2008 , Woolf stated that: "Translational research means different things to different people, but it seems important to almost everyone". ${ }^{2}$ This statement still rings true today, with research funding agencies around the world making translational research a key priority. ${ }^{3-5}$ In Australia, the National Health and Medical Research Council 
has established a translation faculty, funds translation research centres and fellowships, and has implemented a suite of initiatives under the banner of research translation. ${ }^{4}$ One of the challenges in the field of research translation is that interested parties interpret and apply the associated terms in different ways. ${ }^{5}$

For many, the term research translation refers to the 'bench-to-bedside' process, which involves applying knowledge from basic sciences to produce new medicines, devices and treatment options for patients. ${ }^{6,7}$ In this conceptualisation, the interface between basic science and clinical medicine is particularly important, whereby a promising new treatment can be used clinically and commercialised. ${ }^{2}$ For others - such as those in public health and health services research, whose work focuses on healthcare delivery systems and improving population health - the primary outcome is translating research into policy and practice. ${ }^{1,2}$ More recent conceptualisations of research translation have combined these two perspectives of the outcomes of the translation process. ${ }^{6,7}$

Research translation is also often used interchangeably with other related terms, including 'knowledge translation'8, 'knowledge to action' (KTA) ${ }^{9}$ and 'evidence based practice'. ${ }^{10}$ All these terms describe a gap between research knowledge and its application to treatment options, policy and practice, and acknowledge the importance of closing this gap. Regardless of the perspective taken, there is agreement that this process is dynamic, involving interaction between the context, researchers and end users of research knowledge. ${ }^{1,11}$ To measure the success of translation efforts, there is a clear need for conceptual frameworks that can describe and measure the key elements of the research translation process. In light of this, the purpose of this review was to: a) examine different research translation frameworks described in the literature; b) examine the similarities and differences between research translation frameworks; and c) identify key strengths and weaknesses of the models when they are applied in practice.

\section{Methods}

\section{Literature review search strategy}

The review included publications on theoretical frameworks and models that describe processes and issues associated with translation of research evidence into policy and practice. We searched PubMed (MEDLINE and National Center for Biotechnology Information databases) for studies published in English between January 1990 and December 2014, using the search terms (translational research OR knowledge translation OR evidence to practice) AND (framework OR model OR theory) AND (public health OR health promotion OR medicine). Relevant study designs were theoretical and opinion pieces, case studies, descriptive studies and frameworks. Studies were assessed for inclusion by title and abstract, then by full-text review.

'Theories' were defined as ideas that explain research translation and offer principles that translation can be based on. 'Frameworks/models' propose a structure around which research translation can be organised. Frameworks/models are used to inform decisions and judgements about translation, and often apply multiple concepts, processes and theories. Frameworks and models that were highly specific to a health discipline or issue (thus rendering them difficult to apply across health settings) were excluded.

Reference lists of the final papers were also checked to ensure inclusion of further relevant papers in the final review (Figure 1).

\section{Results}

An initial review of 14867 abstracts in PubMed against the inclusion criteria yielded 636 abstracts; searches of the references lists of retrieved papers identified a further 5 documents. Of the 641 full papers and documents reviewed against the inclusion criteria, 98 were included in the review.

\section{Frameworks and models}

Box 1 includes a synthesis of the eight most frequently applied research translation frameworks and models published in two or more papers identified by the search strategy (66 papers). Supplementary table 1 (available from: http://www.health.nsw.gov.au/research/Documents/ supplementary-table-1.pdf) lists the remaining models/ frameworks included in the review.

\section{Study characteristics}

There is a growing body of literature describing frameworks and models for translation of research evidence into practice $-85 \%$ of papers included in the final review were published since 2005. Frameworks included in the review have been applied in myriad settings, including basic and medical sciences, public health, clinical research, disease management, guideline development, global health, environmental health and preventive medicine. These frameworks can successfully support knowledge translation in a number of sectors across health and beyond.

The review identified 41 frameworks and models. By far the most frequently applied knowledge translation framework was the RE-AIM framework developed by Glasgow and colleagues ${ }^{12-28}$, followed by variants of the knowledge translation continuum models or ' $T$ ' models ${ }^{5-7,1129-39}$, the Knowledge to Action framework ${ }^{8,9,38-44}$, the PARiHS framework ${ }^{45-48}$, the evidence based public health models of Brownson and colleagues ${ }^{10,53-57}$, and the stages of research and evaluation model of Nutbeam, Bauman and colleagues. ${ }^{58-62}$ The four most widely 
Figure 1. Literature search process

Included:

Additional papers from reference lists of papers retrieved $(n=5)$

Papers for review of

title and abstract

$(n=14867)$

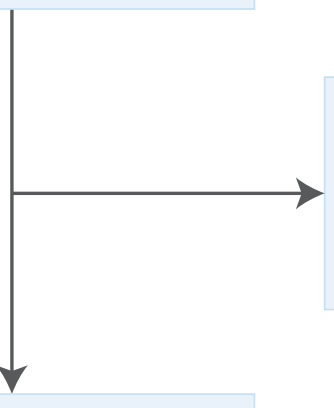

Excluded:

Inclusion criteria not

met $(n=14231)$

Paper for review of

full text $(n=636)$

Excluded:

No discussion of translation framework $(n=506)$

Framework too narrow $(n=37)$

\section{Box 1. Most frequently applied research translation frameworks and models}

\section{RE-AIM}

Number of studies: 17 (citations $^{12-28}$ )

\section{Key elements:}

- Reach (proportion of the target population that participated in the intervention)

- Efficacy or effectiveness (success rate if implemented as in guidelines; defined as positive outcomes minus negative outcomes)
- Adoption (proportion of settings, practices, and plans that will adopt intervention)

- Implementation (extent to which intervention is implemented as intended in the real world)

Major changes over time: Glasgow et al. (1999) ${ }^{12}$ created the initial 5-step framework; then introduced effectiveness in addition to efficacy to improve generalisability (2003). ${ }^{13}$ Dzewaltowski et al. (2004) ${ }^{14}$ considered both individual and organisational levels to better account for local context.

Context applied: Health behaviour change, family medicine, food labelling, patient-centred medical home, falls prevention, community health centres, smoking cessation, implementation grants, diabetes prevention, 
multisector partnerships, burden among dementia caregivers.

Key implications: The model was first developed in 1999 by Glasgow et al. ${ }^{12}$ and revised over the years to better consider external validity by considering effectiveness rather than just efficacy, as well as settinglevel considerations (e.g. organisational vs individual). The RE-AIM framework is useful for estimating public health impact, comparing different health policies, planning policies designed for increased likelihood of success, and identifying areas for integration of policies with other health promotion strategies.

\section{Translation research continuum or ' $T$ ' models}

Number of studies: 14 (citations $^{5-7,11,29-38}$ )

\section{Key elements:}

- Description and discovery

- From discovery to health applications

- From health application to evidence guidelines

- From guidelines to health practice

- Evaluation of effectiveness and cost-effectiveness of such interventions in the real world and in diverse populations.

Major changes over time: Zerhouni $(2003)^{5}$ initiated the T1 (basic science research $\rightarrow$ clinical research) and T2 (clinical research $\rightarrow$ clinical practice) model; Westfall et al. (2007) ${ }^{11}$ introduced T3 (clinical practice $\rightarrow$ large-scale dissemination); Khoury et al. (2010) 6 introduced T4 (largescale dissemination $\rightarrow$ population health outcomes); and Glasgow et al. (2012) introduced T0 (description and discovery). Others have made adaptations of the model to become circular rather than linear (e.g. Callard et al. [2012] ${ }^{34}$ proposed an interlocking loop model).

Context applied: Genomics, child health, breastfeeding, general medical research.

Key implications: Zerhouni et al. (2003) ${ }^{5}$ first proposed the model through the National Institutes of Health Roadmap. The roadmap acknowledges that translational steps are required to realise the potential for medical discoveries at the 'bench' to be applied to better patient care at the 'bedside' and in clinical practice. Originally consisting of two steps, the model has expanded to five including everything between discovery and population health impact. This model can support scaling up of evidence based practices by identifying innovations that are most likely to be effective and successfully implemented.

\section{Knowledge to Action (KTA) framework}

Number of studies: 9 (citations ( $^{8,9,38-44}$ )

Key elements: The KTA framework consists of two interconnected cycles (knowledge creation and action):

- Knowledge creation (in the form of a funnel guided by tailoring knowledge)

- Knowledge inquiry

- Knowledge synthesis

- Knowledge tools/products

- Knowledge creation is linked to action by

- Identifying the problem

- Identifying, reviewing, and selecting knowledge

- Action cycle

- Adapt knowledge to local context

- Assess barriers to knowledge use

- Select, tailor and implement interventions

- Monitor knowledge use

- Evaluate outcomes

- Sustain knowledge use.

Major changes over time: Graham et al. $(2006)^{8}$ first proposed the KTA framework and no significant changes have been made since.

Context applied: Translating knowledge to consumers, critical care nutrition, asthma, osteoporosis, dialysis.

Key implications: The KTA framework provides a conceptual framework for integrating the roles of knowledge creation and knowledge application. The framework can help map the process for translating research evidence into practice, and facilitate selection of appropriate study designs to address barriers, evaluate outcomes and address sustainability.

\section{Promoting Action on Research Implementation in} Health Services (PARiHS) framework

Number of studies: 8 (citations ${ }^{45-52}$ )

Key elements: The framework presents successful research implementation as a function of the relationships among evidence, context and facilitation:

- Evidence

- Research

- Clinical experience

- Patient experience

- Context

- Culture

- Leadership

- Evaluation

- Facilitation

- Purpose 


\section{Role}

- Skills and attributes.

Major changes over time: Kitson, Harvey and McCormack (1998) 45 first proposed the framework; Stetler et al. (2011) $)^{51}$ added more elements to each of the three overarching themes (evidence, context, and facilitation); Bergstrom et al. (2012) $)^{52}$ added context subelements to better consider local context.

Context applied: Postoperative pain management, nursing, neonatal health.

Key implications: The framework argues that successful implementation of research into practice is a function of the interplay of three core elements - level and nature of the evidence; context or environment into which the research is to be placed; and method or way in which the process is facilitated. There is strong consideration of local context and facilitation elements necessary for successful implementation of effective guidelines and policies.

\section{Evidence based public health (EBPH) models}

Number of studies: 6 (citations $^{10,53-57}$ )

\section{Key elements:}

- Community assessment

- Quantifying the issue

- Developing a concise statement of the issue

- Determining what is known through the scientific literature

- Developing and prioritising a policy or program

- Developing plans and implementing interventions

- Evaluating the policy or program.

Major changes over time: Brownson et al. (1999) ${ }^{53}$ first proposed the EBPH model, then added a community assessment step to account for local context (2009). ${ }^{10}$ Small adaptations were made by Jacobs et al. (2012) ${ }^{56}$ and Hess et al. (2014) $)^{57}$.

Context applied: Climate change.

Key implications: EBPH models were designed to help ensure that resources in public health are spent appropriately. The models build on the success of evidence based medicine. Key components of EBPH models include making decisions based on the best available peer-reviewed evidence; using data and information systems systematically; applying programplanning frameworks; and engaging the community. EBPH has emerged as a powerful framework for assessing public health concerns and identifying the most effective health protection strategies.

\section{Stages of research progression (rocket model)}

Number of studies: 6 (citations ${ }^{1,58-62}$ )

\section{Key elements:}

- Problem definition

- Solution generation (program development)

- Intervention testing (process and impact evaluation to determine program efficacy or effectiveness)

- Intervention replication (effective programs are adapted for other settings to determine if similar outcomes can be reproduced)

- Dissemination research (upscaling of a program to a population-wide level).

Major changes over time: Nutbeam and Bauman (2006) ${ }^{58}$ first developed the model; minor adaptations were made by Rychetnik et al. (2012). ${ }^{1}$

Context applied: Falls prevention, public health, child obesity prevention, chronic disease prevention.

Key implications: The rocket model shows the different research and evaluation questions and research methods that are applied in the planning, evaluation and dissemination of a comprehensive set of public health interventions over six stages, and can be used to inform development of public policies and programs. The framework enables decision makers to map the evidence for a given policy or program - that is, to identify what types of evaluations have been done, including translation research, and what of this evidence (if any) informed current policy and practice. The rocket model also provides a useful approach for assessing evidence gaps in health promotion programs, and highlights opportunities to improve the evidence base.

Interactive Systems Framework for Dissemination and Implementation (ISF)

Number of studies: 4 (citations ${ }^{63-66}$ )

Key elements: Three levels:

- Implementing prevention - prevention delivery system (general capacity use, innovation-specific capacity use)

- Supporting the work - prevention support system (general capacity building, innovation-specific capacity building)

- Distilling the information - prevention synthesis and translation system.

These three levels are encapsulated by four pillars:

- Funding

- Macro policy

- Existing research and theory

- Climate.

Major changes over time: None.

Context applied: Teenage pregnancy. 
Key implications: The ISF provides a heuristic for understanding the needs, barriers and resources of the different systems, as well as a structure for summarising existing research and illuminating priority areas for new research and action. The ISF identifies three key systems necessary for bridging research and practice, including the 'synthesis and translation system', the 'support system' and the 'delivery system'. The model recognises the need to synthesise evidence and package the information to better meet the needs of target audiences. The ISF also recognises that the top-down approach to implementation is suboptimal, as implementation efforts require partnerships.

\section{UK Medical Research Council (MRC) framework}

Number of studies: 2 (citations ${ }^{43,67}$ )

Key elements: A cycle consisting of:

- Development (identifying the evidence base)

- Feasibility and piloting (testing procedures)

- Evaluation (assessing effectiveness)

- Implementation (dissemination, surveillance, followup).

Major changes over time: None.

Context applied: Coronary heart disease and depression, osteoporosis.

Key implications: The MRC framework guided the development of interventions that helped improve outcomes in patients with coronary heart disease and depression. It also helped map the process for translating osteoporosis evidence into practice, and facilitated the selection of appropriate study designs to rigorously address barriers, evaluate outcomes and address sustainability.

published frameworks are described in greater detail below (see page 6).

\section{RE-AIM}

The RE-AIM (Reach, Efficacy, Adoption, Implementation and Maintenance) framework has gained great prominence in the field of chronic disease management, and increasingly in public health. This framework assists in the planning, evaluation and reporting of applied research and interventions. ${ }^{12}$ It proposes that it is essential to collect both individual and programlevel measures of institutionalisation (i.e. the extent to which a health promotion practice or policy becomes routine and part of the everyday culture and norms of an organisation). ${ }^{14}$ Table 1 summarises the evaluation dimensions of the RE-AIM framework.

Glasgow and colleagues ${ }^{13}$ argue that reporting and documenting RE-AIM for efficacy trials and effectiveness interventions will help address slow adoption of research into practice because, by its nature, the framework provides information on generalisability and external validity of interventions. There is merit in this argument, as the RE-AIM framework has been successfully applied in numerous real-world case studies. Examples include assessing the translatability of an effective communitybased exercise intervention to prevent falls ${ }^{21}$; examination of factors influencing the impact of a financial incentivebased smoking cessation intervention in a workplace ${ }^{23}$; translation of a computer-assisted diabetes selfmanagement intervention ${ }^{27}$; implementation of a lifestyle intervention tool in primary care ${ }^{17}$; and application to assessing the public health impact of policy change. ${ }^{18}$ In these examples, the RE-AIM framework is used to report on intervention effectiveness, determine the translatability of an intervention, and as an evaluation framework for policies or programs. The framework has also been used to assess the generalisability of published health promotion literature ${ }^{16}$ and to help design and plan public health interventions to improve dissemination. ${ }^{15}$

\section{Research translation continuum models ('T' models)}

Some of the most widely cited translational research models in the literature, with origins in medical research, are the research translation continuum models, or ' $T$ ' models (also known as 'bench-to-beside' models). ${ }^{5}$ Originally proposed in the National Institutes of Health 2003 Roadmap for medical research ${ }^{5}$, they include what the model describes as two major 'research laboratories' where research occurs: the bench (the laboratory) and

Table 1. RE-AIM evaluation dimensions

\begin{tabular}{lc}
\hline Dimension & Level \\
Reach (proportion of target population that participated in the intervention) & Individual \\
\hline Efficacy (success rate if implemented, e.g. in guidelines; defined as positive outcomes minus negative outcomes) & Individual \\
\hline Adoption (proportion of settings, practices and plans that will adopt this intervention) & Organisation \\
\hline Implementation (extent to which intervention is implemented as intended in the real world) & Organisation \\
\hline Maintenance (extent to which program is sustained over time) & Individual and \\
\end{tabular}

$\mathrm{a}$ The product of the five dimensions is the public health impact score (population-based effect)

Source:Dzewaltowski et al. ${ }^{14}$ 
the bedside (in clinical settings). It also includes two translational steps (T1 and T2) and describes how new medical discoveries are translated into clinical practice (T2).

Westfall et al. ${ }^{11}$ proposed an expansion of the model to include an additional research laboratory (practicebased research) and translational step (T3) to improve incorporation of research discoveries into day-to-day clinical care. For example, this stage of translation would focus on ensuring that a new therapy was widely available to those who needed it on a system level or 'in practice'. These 'blue highways' of the roadmap are a continuum, with overlap between sites of research and translational steps. Though this model substantially advances the conceptual development of translation science, it does not acknowledge other important types of research, including community-based participatory research, public health research and health policy analysis.

Building on these 'bench-to-bedside' concepts, another framework with its origins in genomics proposes four phases of translational research: 6

- T1 (translation 1) - basic scientific discovery and its clinical impact on individual health

- T2 - application of evidence based practice guidelines arising from $\mathrm{T} 1$ findings
- T3 - the process of moving guidelines into health practice through delivery and dissemination research

- T4 - the process of translating practice to population health impact.

An example of T4 from Khoury and colleagues ${ }^{6}$ in genomics is that, although public health newborn screening programs have been in place for decades, they have only recently integrated new technologies (particularly tandem mass spectrometry) for identifying an expanded number of disorders. Surveillance and outcomes research are being used to document the real-world effectiveness and potential harms of these new tests. The types of research that characterise each phase include observational and clinical trials in T1; evidence synthesis and guideline development in T2; implementation research and dissemination research in T3; and outcomes research across disciplines and population monitoring in T4. More recently, this model has incorporated an additional precursor stage, T0, in which the problem is identified. ${ }^{7}$

This model extends the 'bench-to-bedside' paradigm by giving due consideration to basic research and clinical practice, and also adding public health approaches to translating scientific discoveries into treatment, prevention and control of human disease in populations. The model

Figure 2. T0-T4 phases of translational research

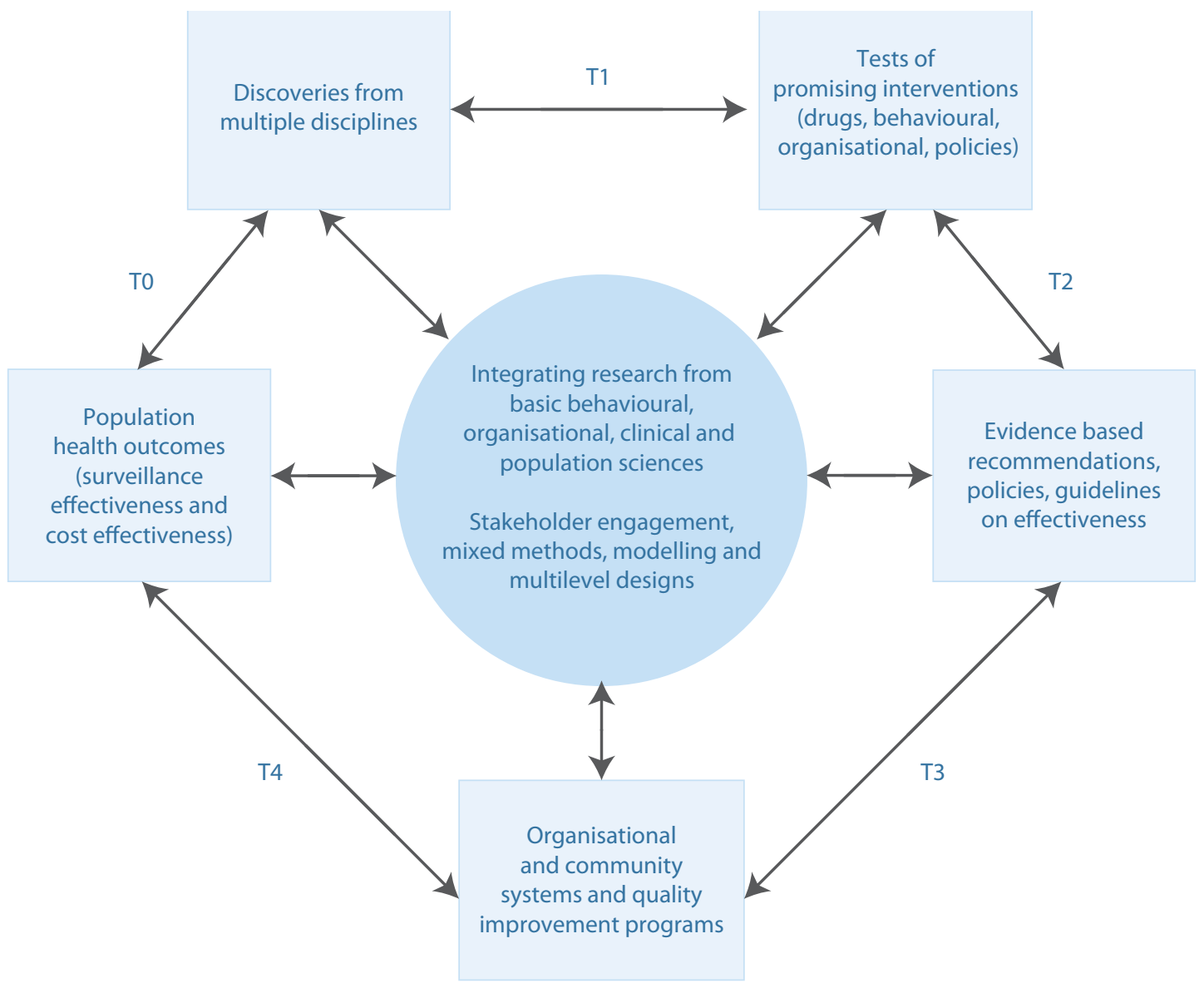


highlights that the true endpoint of translational research is not simply institutionalising effective interventions, but improving population health. Figure 2 shows the relationships across these phases in a further adaption of the model of Khoury and colleagues ${ }^{6}$ by Glasgow and colleagues in $2012 .^{7}$

\section{Knowledge to Action (KTA) framework}

The KTA framework was developed in Canada by Graham and colleagues. ${ }^{8}$ The framework has two components: a 'knowledge creation' funnel and an 'action cycle', each containing multiple phases. Knowledge creation includes knowledge inquiry, knowledge synthesis and the creation of knowledge tools. ${ }^{8}$ The knowledge becomes more refined as it funnels through each stage of the process, resulting in tools for decision making such as clinical practice guidelines or patient decision aids. ${ }^{41}$ The action cycle has seven phases and guides the application of the identified and refined knowledge. The phases can occur sequentially or simultaneously, and the knowledge phases can influence the action phases at any point in the cycle. The elements of the action cycle focus on deliberately bringing about change in healthcare systems and groups. Graham and colleagues describe the phases as "dynamic" and that they "can influence each other". ${ }^{8}$ Knowledge is adapted to the local context, and barriers and facilitators are assessed..$^{9}$ Involvement of stakeholders and tailoring of knowledge to the target audience is critical. ${ }^{9}$ The KTA framework has been used widely to transfer research findings into practice by organisations such as the Canadian Institutes of Health Research. ${ }^{68}$

Figure 3. Knowledge to Action (KTA) framework

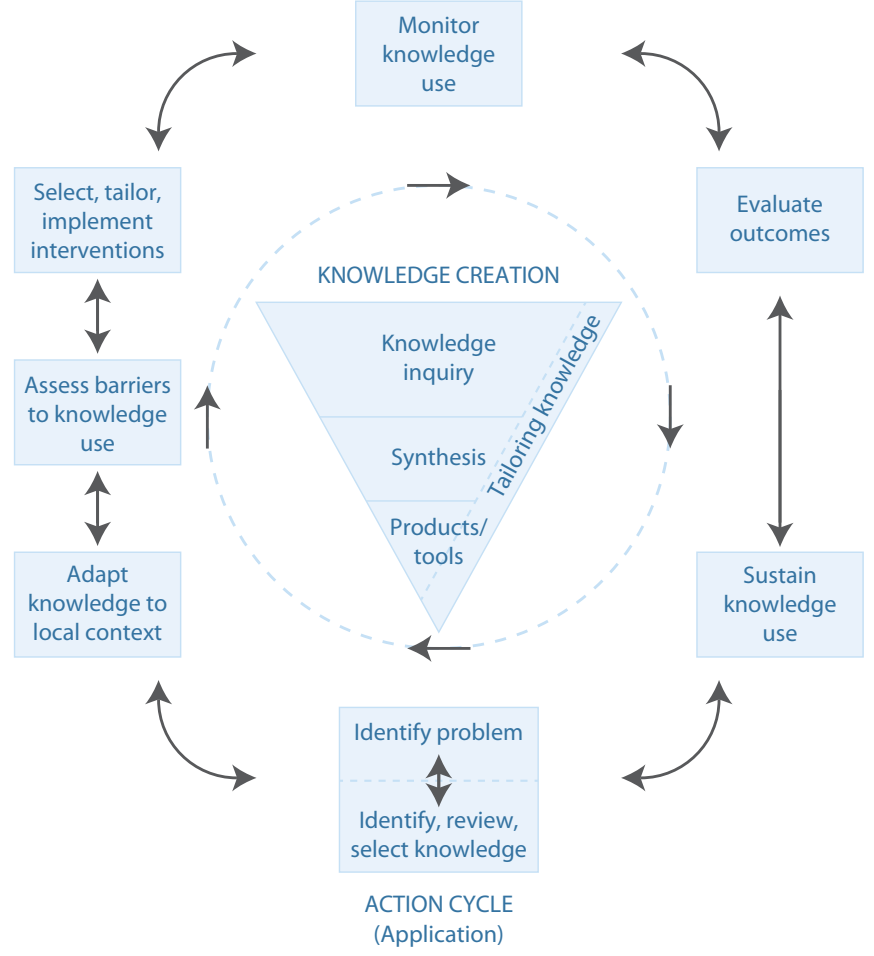

\section{Promoting Action on Research Implementation in Health Services (PARiHS) framework}

This framework was first developed by Kitson, Harvey and McCormack in $1998^{45}$ and the term 'PARiHS' was coined by Rycroft-Malone and colleagues in $2002 .{ }^{46}$ It proposes that successful implementation of research into practice is a function of the interplay between three elements: the nature of the evidence, the context in which the proposed change is to be implemented, and the mechanism by which change is facilitated. ${ }^{47}$ The framework considers these elements to have a dynamic relationship, and each is positioned on a 'high to low' continuum. ${ }^{47}$ For example, an intervention that is developed based on strong evidence would be rated 'high' in the evidence element. The framework proposes that, for implementation to be successful, there needs to be clarity about the nature of the evidence used, the quality of the context, and the type of facilitation needed to ensure a successful change process. ${ }^{47}$ The PARiHS framework has supported knowledge translation in a variety of settings, including pain management, nursing practices and neonatal health. ${ }^{46-48}$ There is a growing body of evidence showing the PARiHS framework has conceptual integrity, and face and conceptual validity. ${ }^{69}$

Figure 4. Promoting Action on Research Implementation in Health Services (PARiHS) framework

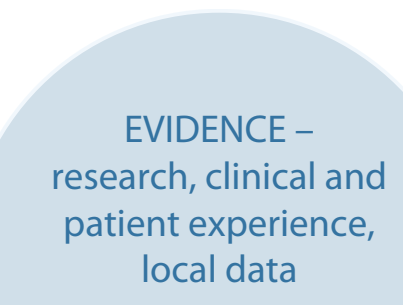

CONTEXT -

culture,

leadership,

evaluation
FACILITATION purpose, role, skills and attributes 


\section{Discussion}

\section{Similarities and differences between the models}

All these frameworks describe a gap between research knowledge and its application to treatment options, policy and practice, and acknowledge the importance of closing this gap. They all articulate processes for applying evidence from research to intervention development, then applying interventions with demonstrated efficacy in controlled environments into new settings with different populations, and ultimately disseminating effective interventions into policy and practice.

Of note, the most frequently applied research translation models and frameworks identified in this review each stem from different fields, including implementation science (RE-AIM), basic and medical sciences ('T' models), health services research (KTA and PARiHS frameworks) and public health (EBPH and research progression models). It is therefore not surprising that these models appear to lend themselves more readily to practical application in these respective fields.

It is clear from this review that the term 'research translation' is often used interchangeably with other related terms such as 'knowledge translation', 'knowledge to action', 'evidence based policy and practice' and 'research implementation'. This lack of consistent terminology can be a source of confusion. The concept of research translation in particular is increasingly recognised internationally as an important function of academia and is a growing priority for major healthrelated funders. ${ }^{3-5}$ However, for this field to move forward in a coherent way, a common language is needed to describe the processes and types of research associated with translation of research evidence into broader policy and practice.

All these frameworks acknowledge the difficulty of closing the gap between research and practice, and that this gap is in part caused by a failure of research to meet the information needs of policy makers and practitioners; characteristics of the intervention (particularly if it is overly intensive, expensive and difficult to implement in other populations and settings); and the research or evaluation designs giving insufficient attention to real-world contexts and the complex interaction of these factors.

\section{Strengths and weaknesses of the models when applied in practice}

The evidence based public health framework proposed by Brownson and colleagues ${ }^{10}$ offers a practical evidence-to-practice approach and, in more recent iterations, acknowledges the importance of contextual implementation factors. The 'bench-to-bedside' approach of the National Institutes of Health Roadmap ${ }^{7}$ and the later iteration by Westfall et al. ${ }^{11}$ provide a detailed translation process that captures the types of research needed to influence practice, but they do not adequately describe the processes required for wider adoption and dissemination of research evidence into systems. Frameworks for translation of bench science into clinical practice ('T' models), particularly in their earlier incarnations, had limited practical public health application due to lack of attention to population health outcomes and system impacts. Despite this, they are the most widely applied models by health funding bodies, particularly in the US and Australia. This drives public health and clinical researchers to use these narratives in describing translation efforts, even though they sit more naturally with basic and clinical sciences. It could be reasonably argued that this places public health researchers at a disadvantage, particularly because many of their translation outcomes are more distal than those of clinical researchers.

However, it is important to acknowledge that the addition of a T4 stage by Khoury and colleagues ${ }^{6}$ in their 'translational epidemiology' model is a substantial improvement on previous variants of the ' $T$ ' model because it focuses real-world effectiveness at the population or healthcare-system level. Encouragingly, there seems to be increasing recognition that the endpoint of translation is broader than clinical practice, and that it includes population health and system change. Interestingly, the only other models to have similar stages are the maintenance phase in RE-AIM ${ }^{12}$ and the sixth program monitoring phase in the Nutbeam and Bauman model. ${ }^{58}$ Importantly, these advances move beyond dissemination and stand-alone evaluation to a process for monitoring system change.

Although RE-AIM provides a comprehensive and well-tested translation framework, there are a number of challenges to its practical application. The first is the expression of efficacy as positive outcomes minus negative outcomes. This oversimplifies the process of assessing the efficacy of an intervention, and is problematic because it is very difficult to quantify positive outcomes in a way that can be directly compared with negative outcomes. In addition, it does not give sufficient consideration to monitoring and reporting contextual factors that support or hinder the adoption and dissemination of promising interventions into broader policy and practice.

The PARiHS framework came to prominence because of its explicit consideration of contextual factors as a critical aspect of successful implementation of programs and policies. By treating evidence, context and facilitation as equally important in research translation, the framework supports researchers and policy makers to understand the full range of factors affecting translation of evidence into practice. The most recent revision of the model by Bergström et al. ${ }^{52}$ proposed the inclusion of a greater number of context subelements, further demonstrating an increasing focus on contextual factors. 
The KTA framework is one of the few models not to have undergone significant revision since being developed in 2006. This is because Graham and colleagues $^{8}$ recognised that research translation is a continuous, cyclic process that requires continuous refinement over time. The framework acknowledges that research translation may not necessarily follow a pipeline model, and that each element in the translation process influences other elements. It also recognises that knowledge is constantly evolving and can feed into policy making at various stages. Finally, the model acknowledges that research evidence has to be adapted to the local context to be useful in informing policy and practice. As a result, the model has been highly successful and adopted as a national research translation framework in Canada. ${ }^{69}$

All these models would benefit from more detailed consideration of the processes associated with scaling up promising interventions within complex community and organisational contexts. Notably, none of the reviewed models specifically addresses costs. Cost, cost effectiveness and cost benefit are often key factors in determining how widely adopted an intervention will be in practice, and could figure more prominently in these models.

Despite its potential usefulness as a conceptual model, the Nutbeam and Bauman model is yet to be widely tested using real-world case studies in policy and practice. This may be partly explained by the fact that the model is a relatively new arrival, first published in 2006. In contrast, the most commonly applied model in the literature, RE-AIM, was first published in the peerreviewed literature in 1999, allowing more time for the model and associated concepts to permeate thinking in the field.

\section{Areas for further research}

It is important to consider the comparative effectiveness of these frameworks in supporting the development and implementation of successful programs and policies. All the frameworks in this review have the common goal of bridging the gap between evidence and practice. Understanding which is most effective and in what contexts will support the development of better policies and programs that have the greatest impact on improving population health.

Further, more work is needed to determine how research translation frameworks are being used by researchers, policy makers and institutions. This review has identified a number of published case studies, all of which demonstrated successful application of research translation frameworks. We encourage authors to document accounts of successful and unsuccessful application of these frameworks in real-world case studies and, importantly, encourage journals to publish these data. To comprehensively investigate the relative success of the application of these frameworks, further research involving document analysis and interviews with users of these frameworks is recommended. A better understanding of how research translation frameworks are being used in health policy and program development will allow us to better identify the key challenges to effective research translation and assist in understanding the role that these frameworks can potentially play in bridging the evidence-practice gap.

\section{Limitations of the review}

A single database was searched for this review, which may mean that papers were missed. However, PubMed is a large database, so it is likely that the most relevant frameworks and models were identified. The study applied a systematic review methodology with narrative synthesis, which differs from approaches used in Cochrane reviews. This decision was made as the topic under consideration was better suited to narrative analysis.

\section{Conclusion}

Conceptual models for research translation are interpreted and applied by different health fields in different but related ways. All of the reviewed models acknowledge a gap between research knowledge and its application to treatment options, policy and practice, and propose pathways to closing this gap. All the models articulate processes of applying evidence from research to intervention development, then applying interventions with demonstrated efficacy into new settings with different populations, and ultimately disseminating effective interventions into policy and practice.

\section{Acknowledgements}

The authors thank Dr Michael Giffin for editing the manuscript.

\section{Competing interests}

None declared

\section{Author contributions}

AM developed the concept for the paper, framed the search strategy, categorised papers and led the manuscript production. BL conducted the literature search, conducted the paper extraction, assessed and categorised papers, and contributed to drafting and editing the manuscript. 


\section{References}

1. Rychetnik L, Bauman A, Laws R, King L, Rissel C, Nutbeam D, et al. Translating research for evidencebased public health: key concepts and future directions. J Epidemiol Community Health. 2012;66(12):1187-92.

2. Woolf $\mathrm{SH}$. The meaning of translational research and why it matters. JAMA. 2008;299(2):211-13.

3. NIH. National Eye Institute (NEI). Research today ... vision tomorrow. Bethesda, MD: National Institutes of Health. NIH roadmap for medical research; [cited 2016 Sep 7] [about 3 screens]. Available from: nei.nih. gov/funding/roadmap

4. Australian Government. National Health and Medical Research Council. Canberra: NHMRC; 2016. Advanced health research and translation centres; 2016 [cited 2016 Sep 7] [about 4 screens]. Available from: www. nhmrc.gov.au/research/nhmrc-advanced-health-researchand-translation-centres

5. Zerhouni E. Medicine. The NIH roadmap. Science. 2003;302(5642):63-72.

6. Khoury MJ, Gwinn M, loannidis JPA. The emergence of translational epidemiology: from scientific discovery to population health impact. Am J Epidemiol. 2010;172(5):517-24.

7. Glasgow RE, Vinson C, Chambers D, Khoury MJ, Kaplan RM, Hunter C. National Institutes of Health approaches to dissemination and implementation science: current and future directions. Am J Public Health. 2012;102(7):1274-81.

8. Graham ID, Logan J, Harrison MB, Straus SE, Tetroe J, Caswell W, Robinsons N. Lost in knowledge translation: time for a map? J Contin Educ Health Prof. 2006;26(1):13-24.

9. Field B, Booth A, Ilott I, Gerrish K. Using the Knowledge to Action Framework in practice: a citation analysis and systematic review. Implement Sci. 2014;9:172.

10. Brownson RC, Fielding JE, Maylahn CM. Evidence-based public health: a fundamental concept for public health practice. Annu Rev Public Health. 2009;30:175-201.

11. Westfall JM, Mold J, Fagnan L. Practice-based research--"blue highways" on the NIH roadmap. JAMA. 2007;297(4):403-6.

12. Glasgow RE, Vogt TM, Boles SM. Evaluating the public health impact of health promotion interventions: the REAIM framework. Am J Public Health. 1999;89(9):1322-7.

13. Glasgow RE, Lichtenstein E, Marcus AC. Why don't we see more translation of health promotion research to practice? Rethinking the efficacy-to-effectiveness transition. Am J Public Health. 2003;93(8):1261-7.

14. Dzewaltowski DA, Glasgow RE, Klesges LM, Estabrooks PA, Brock E. RE-AIM: evidence-based standards and a web resource to improve translation of research into practice. Ann Behav Med. 2004;28(2):7580.
15. Klesges LM, Estabrooks PA, Dzewaltowski DA, Bull SS, Glasgow RE. Beginning with the application in mind: designing and planning health behavior change interventions to enhance dissemination. Ann Behav Med. 2005;29 Suppl:66-75.

16. Glasgow RE, Klesges LM, Dzewaltowski DA, Estabrooks PA, Vogt TM. Evaluating the impact of health promotion programs: using the RE-AIM framework to form summary measures for decision making involving complex issues. Health Educ Res. 2006;21(5):688-94.

17. Glasgow RE. RE-AIMing research for application: ways to improve evidence for family medicine. J Am Board Fam Med. 2006;19(1):11-19.

18. Jilcott S, Ammerman A, Sommers J, Glasgow RE. Applying the RE-AIM framework to assess the public health impact of policy change. Ann Behav Med. 2007;34(2):105-14.

19. Bakken S, Ruland CM. Translating clinical informatics interventions into routine clinical care: how can the RE-AIM framework help? J Am Med Inform Assoc. 2009;16(6):889-97.

20. Glasgow RE, Dickinson P, Fisher L, Christiansen S, Toobert DJ, Bender BG, et al. Use of RE-AIM to develop a multi-media facilitation tool for the patient-centered medical home. Implement Sci. 2011;6:118.

21. Shubert TE, Altpeter M, Busby-Whitehead J. Using the RE-AIM framework to translate a research-based falls prevention intervention into a community-based program: lessons learned. J Safety Res. 2011;42(6):509-16.

22. Weiss SM, Jones DL, Lopez M, Villar-Loubet O, Chitalu N. The many faces of translational research: a tale of two studies. Transl Behav Med. 2011;1(2):327-30.

23. Kim AE, Towers A, Renaud J, Zhou J, Shea JA, Galvin R, Volpp KG. Application of the RE-AIM framework to evaluate the impact of a worksite-based financial incentive intervention for smoking cessation. J Occup Environ Med. 2012;54(5):610-14.

24. Kessler RS, Purcell EP, Glasgow RE, Klesges LM, Benkeser RM, Peek CJ. What does it mean to "employ" the RE-AIM model? Eval Health Prof. 2013;36(1):44-66.

25. Almeida FA, Pardo KA, Seidel RW, Davy BM, You W, Wall SS, et al. Design and methods of "diaBEAT-it!": a hybrid preference/randomized control trial design using the RE-AIM framework. Contemp Clin Trials. 2014;38(2):383-96.

26. Sweet SN, Ginis KAM, Estabrooks PA, LatimerCheung AE. Operationalizing the RE-AIM framework to evaluate the impact of multi-sector partnerships. Implement Sci. 2014;9:74.

27. Matthews L, Kirk A, Macmillan F, Mutrie N. Can physical activity interventions for adults with type 2 diabetes be translated into practice settings? A systematic review using the RE-AIM framework. Transl Behav Med. 2014;4(1):60-78 
28. Altpeter M, Gwyther LP, Kennedy SR, Patterson TR, Derence K. From evidence to practice: using the RE-AIM framework to adapt the REACHII caregiver intervention to the community. Dementia (London). 2015;14(1):104-13.

29. Khoury MJ, Gwinn M, Yoon PW, Dowling N, Moore CA, Bradley $L$. The continuum of translation research in genomic medicine: how can we accelerate the appropriate integration of human genome discoveries into health care and disease prevention? Genet Med. 2007;9(10):665-74.

30. Dougherty D, Conway PH. The "3T's" road map to transform US health care: the "how" of high-quality care. JAMA. 2008;299(19):2319-21.

31. Fleming ES, Perkins J, Easa D, Conde JG, Baker RS, Southerland WM, et al. The role of translational research in addressing health disparities: a conceptual framework. Ethn Dis. 2008;18(2 Suppl 2):S2-155-60.

32. Drolet BC, Lorenzi NM. Translational research: understanding the continuum from bench to bedside. Transl Res. 2011;157(1):1-5.

33. Bergman DA, Beck A. Moving from research to largescale change in child health care. Acad Pediatr. 2011;11(5):360-8.

34. Callard F, Rose D, Wykes T. Close to the bench as well as at the bedside: involving service users in all phases of translational research. Health Expect. 2012;15(4):389400.

35. Spoth R, Rohrbach LA, Greenberg M, Leaf $P$, Brown $C H$, Faga $A$, et al. Addressing core challenges for the next generation of type 2 translation research and systems: the translation science to population impact (TSci Impact) framework. Prev Sci. 2013;14(4):319-51.

36. Rubio DM, Robinson GF, Gilliam VA, Primack BA, Switzer GE, Seltzer DL, Kapoor WN. Characterization of investigators' approach to translational research: a qualitative study. Clin Transl Sci. 2014;7(6):441-6.

37. Atchan M, Davis D, Foureur M. Applying a knowledge translation model to the uptake of the Baby Friendly Health Initiative in the Australian health care system. Women Birth. 2014;27(2):79-85.

38. Tugwell PS, Santesso NA, O'Connor AM, Wilson AJ, Effective Consumer Investigative Group. Knowledge translation for effective consumers. Phys Ther. 2007;87(12):1728-38.

39. Straus SE, Tetroe J, Graham I. Defining knowledge translation. CMAJ. 2009;181(3-4):165-8.

40. Heyland DK, Cahill NE, Dhaliwal R. Lost in (knowledge) translation! JPEN J Parenter Enteral Nutr. 34(6):610-15.

41. Straus SE, Tetroe JM, Graham ID. Knowledge translation is the use of knowledge in health care decision making. J Clin Epidemiol. 2011;64(1):6-10.
42. Licskai C, Sands T, Ong M, Paolatto L, Nicoletti I. Using a knowledge translation framework to implement asthma clinical practice guidelines in primary care. Int J Qual Health Care. 2012;24(5):538-46.

43. Kastner M, Straus SE. Application of the Knowledge-toAction and Medical Research Council frameworks in the development of an osteoporosis clinical decision support tool. J Clin Epidemiol. 2012;65(11):1163-70.

44. Sood MM, Manns B, Nesrallah G. Using the knowledgeto-action framework to guide the timing of dialysis initiation. Curr Opin Nephrol Hypertens. 2014;23(3):321-7.

45. Kitson A, Harvey G, McCormack B. Enabling the implementation of evidence based practice: a conceptual framework. Qual Health Care. 1998;7(3):149-58.

46. Rycroft-Malone J, Harvey G, Kitson A, McCormack B, Seers K, Titchen A. Getting evidence into practice: ingredients for change. Nurs Stand. 2002;16(37):38-43.

47. Rycroft-Malone J. The PARIHS framework--a framework for guiding the implementation of evidence-based practice. J Nurs Care Qual. 2004;19(4):297-304.

48. Brown D, McCormack B. Developing postoperative pain management: utilising the promoting action on research implementation in health services (PARIHS) framework. Worldviews Evid Based Nurs. 2005;2(3):131-41.

49. Ellis I, Howard P, Larson A, Robertson J. From workshop to work practice: an exploration of context and facilitation in the development of evidence-based practice. Worldviews Evid Based Nurs. 2005;2(2):84-93.

50. Doran DM, Sidani S. Outcomes-focused knowledge translation: a framework for knowledge translation and patient outcomes improvement. Worldviews Evid Based Nurs. 2007;4(1):3-13.

51. Stetler CB, Damschroder LJ, Helfrich CD, Hagedorn HJ. A guide for applying a revised version of the PARIHS framework for implementation. Implement Sci. 2011;6:99.

52. Bergström A, Peterson S, Namusoko S, Waiswa P, Wallin L. Knowledge translation in Uganda: a qualitative study of Ugandan midwives' and managers' perceived relevance of the sub-elements of the context cornerstone in the PARIHS framework. Implement Sci. 2012;7:117.

53. Brownson RC, Gurney JG, Land GH. Evidencebased decision making in public health. J Public Health Manag Pract. 1999;5(5):86-97.

54. Kohatsu ND, Robinson JG, Torner JC. Evidence-based public health: an evolving concept. Am J Prev Med. 2004;27(5):417-21.

55. McGuire WL. Beyond EBM: new directions for evidencebased public health. Perspect Biol Med. 2005;48(4):55769.

56. Jacobs JA, Jones E, Gabella BA, Spring B, Brownson RC. Tools for implementing an evidence-based approach in public health practice. Prev Chronic Dis. 2012;9:E116. 
57. Hess JJ, Eidson M, Tlumak JE, Raab KK, Luber G. An evidence-based public health approach to climate change adaptation. Environ Health Perspect. 2014;122(11):1177-86.

58. Bauman A, Nutbeam D. Evaluation in a nutshell: a practical guide to the evaluation of health promotion programs. Southampton: University of Southampton; 2013 [cited 2016 May 30]. Available from: http://eprints. soton.ac.uk/359818/

59. Milat AJ, Monger C, Smith J, Bauman A, Redman S, Goodger B. The strategic development of the NSW health plan for prevention of falls and harm from falls among older people: 2011-2015; translating research into policy and practice. N S W Public Health Bull. 2011;22(3-4):737.

60. Milat AJ, Bauman AE, Redman S, Curac N. Public health research outputs from efficacy to dissemination: a bibliometric analysis. BMC Public Health. 2011;11:934.

61. Rissel C, Laws R, St George A, Hector D, Milat AJ, Baur LA. Research to practice: application of an evidence-building framework to a childhood obesity prevention initiative in New South Wales. Health Promot J Austr. 2012;23(1):16-24

62. O'Hara BJ, Phongsavan P, King L, Develin E, Milat AJ, Eggins D, et al. 'Translational formative evaluation': critical in up-scaling public health programmes. Health Promot Int. 2014;29(1):38-46.

63. Wandersman A, Duffy J, Flaspohler P, Noonan R, Lubell K, Stillman L, et al. Bridging the gap between prevention research and practice: the interactive systems framework for dissemination and implementation. Am J Community Psychol. 2008;41(3-4):171-81.
64. Lesesne CA, Lewis KM, White CP, Green DC, Duffy JL, Wandersman A. Promoting science-based approaches to teen pregnancy prevention: proactively engaging the three systems of the interactive systems framework. Am J Communit Psychol. 2008;41(3-4):379-92.

65. Flaspohler P, Lesesne CA, Puddy RW, Smith E, Wandersman A. Advances in bridging research and practice: introduction to the second special issue on the interactive system framework for dissemination and implementation. Am J Community Psychol. 2012;50(34):271-81.

66. Chambers DA. The interactive systems framework for dissemination and implementation: enhancing the opportunity for implementation science. Am J Community Psychol. 2012;50(3-4):282-4.

67. Barley EA, Haddad M, Simmonds R, Fortune Z, Walters P, Murray J, et al. The UPBEAT depression and coronary heart disease programme: using the UK Medical Research Council framework to design a nurse-led complex intervention for use in primary care. BMC Fam Pract. 2012;13:119.

68. Government of Canada. Canadian Institutes of Health Research. Ottowa: Canadian Institues of Health Research; 2016. Knowledge translation in health care: moving from evidence to practice; 2015 Nov 18 [cited 2016 Jun 7] [about 4 screens]. Available from: www.cihrirsc.gc.ca/e/40618.html.

69. Kitson AL, Rycroft-Malone J, Harvey G, McCormack B, Seers K, Titchen A. Evaluating the successful implementation of evidence into practice using the PARiHS framework: theoretical and practical challenges. Implement Sci. 2008;3:1.

\section{Copyright: (c) (i) (2)}

(C) 2017 Milat and Li. This article is licensed under the Creative Commons Attribution-NonCommercial-ShareAlike 4.0 International Licence, which allows others to redistribute, adapt and share this work non-commercially provided they attribute the work and any adapted version of it is distributed under the same Creative Commons licence terms. See: www.creativecommons.org/licenses/by-nc-sa/4.0/ 